\title{
Optical System Design of Inter-Spacecraft Laser Interferometry Telescope
}

\author{
Shengnan Chen ${ }^{1}$, Huilin Jiang1 ${ }^{*}$, Chunyan Wang ${ }^{1}$, Zhe Chen ${ }^{2}$ \\ ${ }^{1}$ Department of Optical Engineering, Changchun University of Science and Technology, Changchun, China \\ ${ }^{2}$ Changchun Institute of Optics and Fine Mechanics and Physics, Chinese Academy of Sciences, Changchun, China \\ Email: *865666068@qq.com
}

How to cite this paper: Chen, S.N., Jiang, H.L., Wang, C.Y. and Chen, Z. (2019) Optical System Design of Inter-Spacecraft Laser Interferometry Telescope. Optics and Photonics Journal, 9, 26-37. https://doi.org/10.4236/opj.2019.98B004

Received: July 12, 2019

Accepted: August 6, 2019

Published: August 9, 2019

\begin{abstract}
The fundamental measurement of space gravitational wave detection is to monitor the relative motion between pairs of freely falling test masses using heterodyne laser interferometry to a precision of $10 \mathrm{pm}$. The masses under test are millions of kilometers apart. The inter-spacecraft laser interferometry telescope deliver laser efficiently from one spacecraft to another. It is an important component of the gravitational wave detection observatory. It needs to meet the requirements of large compression ratio, high image quality and extraordinary stray light suppression ability. Based on the primary aberration theory, the method of the large compression ratio off-axis four-mirror optical system design is explored. After optimization, the system has an entrance pupil of $200 \mathrm{~mm}$, compression ratio of 40 times, scientific field of view (FOV) of $\pm 8 \mu \mathrm{rad}$. To facilitate suppressing the stray light and delivering the laser beam to the back-end scientific interferometers, the intermediate images and the real exit pupils are spatially available. Over the full FOV, the maximum root mean square (RMS) wavefront error is less than $0.007 \lambda$, PV value is less than $0.03 \lambda(\lambda=1064 \mathrm{~nm})$. The image quality is approached to the diffraction-limit. The TTL noise caused by the wavefront error of the telescope is analyzed. The TTL noise in the image space of $300 \mu \mathrm{rad}$ range is less than $1 \times 10^{-10} \mathrm{~m}$ whose slope is lower than $0.6 \mu \mathrm{m} / \mathrm{rad}$, which is under the noise budget of the laser interferometer space antenna (LISA), satisfying the requirements of space gravitational wave detection.
\end{abstract}

\section{Keywords}

Laser Interferometry, Telescope, Optical Design, Gravitational Waves

\section{General Instructions}

The spectrum of gravitational waves is a function of frequency and time. Gravi- 
tational waves in the mid-low frequency range of $0.1 \mathrm{mHz}$ to $1.0 \mathrm{~Hz}$ contain abundant information from the early structure and evolution of the universe. Space-based gravitational wave detection can break through the limits of ground measurement scales and establish a measurement baseline at gigameter scale, thereby improving the of detection sensitivity. In addition, it can effectively avoid the effects of ground-surface vibration and gravity gradient noise. It enables the gravitational waves detection in the medium and low frequency range, complements the frequency band of the ground gravitational wave detection.

Unlike the homodyne interferometry technology used in ground-basesd gravitational wave detection, the space-based gravitational wave detection takes advantage of the Doppler shift generated by the relative motion of the spacecraft, uses heterodyne interferometry method to measure the distance between the freely falling masses, and finally computes the gravitational wave signal.

By far, many space-based gravitational wave detection missions have been established, including the laser interferometer space antenna of the European Space Agency (ESA) [1] and Taiji Program in Space of the Chinese Academy of Sciences [2], etc. Among the observatory missions above, similar technical route is adopted. The gravitational wave observatory under design consists of three spacecraft that form an equilateral triangle with an arm length in the range of millions of kilometers that follow Earth in a heliocentric orbit. Each spacecraft consists of two optical telescopes, two inertial sensors and an optical bench. When the gravitational wave passes through the constellation of the gravitational wave observatory, the distance between the test masses at the apex of the triangle alters periodically, and this relative motion is monitored by heterodyne laser interferometry to measure the gravitational wave signal.

The optical telescope [3] is designed to accomplish the transmission and reception of laser beams between two spacecraft with a distance of several millions kilometers: after collimated and expanded the laser beam is send out by the telescope; at the other end of the measurement arm, the far-field diffraction plane wavefront is cut out by the entrance pupil of the local telescope and after compression the beam is delivered into the native scientific interferometer. The gravitational wave signal detection task is high-demanding on the noise level and precision of the Space laser interferometry system. The intensity and phase distribution of the transmitted and received signals are affected directly by the wavefront quality and stability, which is essential for the accuracy of the gravitational wave measurement.

The optical design of the telescope should avoid the employment of refractive components to eliminate temperature effect on the refractive index. Since the length of the measurement arm is in the range of millions of kilometers, the telescope should have large compression ratio, which means, it requires enough entrance aperture to ensure that the received signal has enough intensity, and at the same time the exit pupil is as small as the local laser beam and detector, so that the measurement beam and the reference beam can be superimpose effec- 
tively. Stray light in the telescope, especially backscattered light, is one of the main sources of measurement noise. In order to meet the above requirements, the optical system of the inter-spacecraft laser interferometry telescope generally adopts four-mirror optical structure. The coaxial four-mirror system generally has better resistance to environmental effects, but the on-axis obscuration and secondary mirror causes unacceptably high levels of scattered light and wavefront imperfection. In this paper, an off-axis four-mirror afocal optical structure with characteristics of large compression ratio and high stray light specification is optimized, satisfying the design requirements.

The total noise of the space-based heterodyne laser interferometry system should be lower than $8 \mathrm{pm} / \sqrt{\mathrm{Hz}}$. The angular misalignment of the measuring beam and reference beam will coupled into the optical path result of interferometry measurement to produce tilt-to-length (TTL) noise [4]. As one of the major noise sources in space gravitational wave detection, the Albert Einstein Institution (AEI) in Germany did substantive research about TTL coupling [5], including its generation mechanism and influencing factors, but the research mostly concentrated in the field of interferometer design and analysis [6]. The TTL noise originated from wavefront error and other factors of the telescope also couples into the scientific interferometer and becomes a significant part of measurement noise. Based on the optical design of the telescope, the TTL noise generated by the wavefront residual is analyzed and evaluated.

\section{Requirements}

In order to meet the application of space gravitational wave detection, the key requirements of the telescope design are shown in Table 1 . The operating wavelength of the telescope is determined by the laser used in the interferometry system. The laser source needs to have a short wavelength to reduce the energy loss due to diffraction effects, and have excellent frequency and amplitude stability. The field of regard refers to the field of view that a valid inter-spacecraft laser link can be establish in, namely, the field of view spatial acquisition can be accomplished. However, under the influence of the long-distance arm and the limited speed of light, considering the platform stability and pointing accuracy, the boresight of the telescope on the receiving end should be located within the scientific field of view of the telescope on the transmitting end to complete the

Table 1. Optical system requirements.

\begin{tabular}{cc}
\hline Requirements & Parameters \\
\hline Optical aperture & $200 \mathrm{~mm}$ \\
Operating wavelength & $1064 \mathrm{~nm}$ \\
Field-of-Regard & $\pm 200 \mu \mathrm{rad}$ \\
Scientific field of view & $\pm 8 \mu \mathrm{rad}$ \\
Laser beam compression ratio & 40 \\
Wavefront Error (in scientific FOV) & $\leq \lambda / 30 \mathrm{RMS}(\lambda=1064 \mathrm{~nm})$
\end{tabular}


gravitational wave measurement. With the jitter of the boresight between the two spacecraft in the heliocentric orbit, the asymmetric wavefront error of the telescope will eventually lead to the generation of TTL noise. In addition, in order to ensure the energy transfer efficiency during signal propagation, and to reserve margin for manufacture and assembly, the design residuals should be minimized.

\section{Off-Axis Four Anti-Focus Optical System Design Method}

Based on the coaxial four-mirror optical structure [7], the off-axis four-mirror system can be obtained by optimized after introducing an offset into the coaxial apertures [8]. The system consists of primary mirror $M_{1}$, secondary mirror $M_{2}$, tertiary mirror $M_{3}$ and quaternary mirror $M_{4}$. In order to ensure that the received signal propagate onto the phase meter of the interferometer, the optical system should have a real exit pupil located after the Quaternary mirror. In addition, considering synthetically on the influence of stray light specification and wavefront error, an intermediate image plane should be set between Secondary mirror and Tertiary mirror to facilitate the design and optimization of the field stop and stray light shield. The initial structure of the telescope optical system is shown in Figure 1. We define $h_{\mathrm{i}}$ as the incident height of the edge ray on $\mathrm{M}_{\mathrm{i}}, l_{\mathrm{i}}$ as the object distance of $M_{i}, l_{i}$ as the image distance of $M_{i}, u_{i}$ as the aperture angle of $\mathrm{M}_{\mathrm{i}}$ in object space, $u_{\mathrm{i}}$ as the aperture angle of $\mathrm{M}_{\mathrm{i}}$ in image space, $D_{\mathrm{i}}$ is the space between $\mathrm{M}_{\mathrm{i}}$ and $\mathrm{M}_{\mathrm{i}+1}, R_{\mathrm{i}}$ as the radius of curvature of $\mathrm{M}_{\mathrm{i}}, y_{\mathrm{i}}$ is the height of the cheif ray at $\mathrm{M}_{\mathrm{i}}$, and $k_{\mathrm{i}}$ is the conic coefficient of $\mathrm{M}_{\mathrm{i}}$. The structural parameters include the obscuration ratio of the secondary mirror to the primary mirror $\alpha_{1}$, the tertiary mirror to the secondary mirror $\alpha_{2}$, the quaternary mirror to the tertiary mirror $\alpha_{3}$, and the magnification of the secondary mirror $\beta_{1}$ and the tertiary mirror $\beta_{2}$. The structural parameters satisfy the following relationship:

$$
\begin{aligned}
& \alpha_{1}=l_{2} / f_{1}^{\prime} \approx h_{2} / h_{1} \\
& \alpha_{2}=l_{3} / l_{2}^{\prime} \approx h_{3} / h_{2} \\
& \alpha_{3}=l_{4} / l_{3}^{\prime} \approx h_{4} / h_{3} \\
& \beta_{1}=l_{2}^{\prime} / l_{2}=u_{2} / u_{2}^{\prime}
\end{aligned}
$$

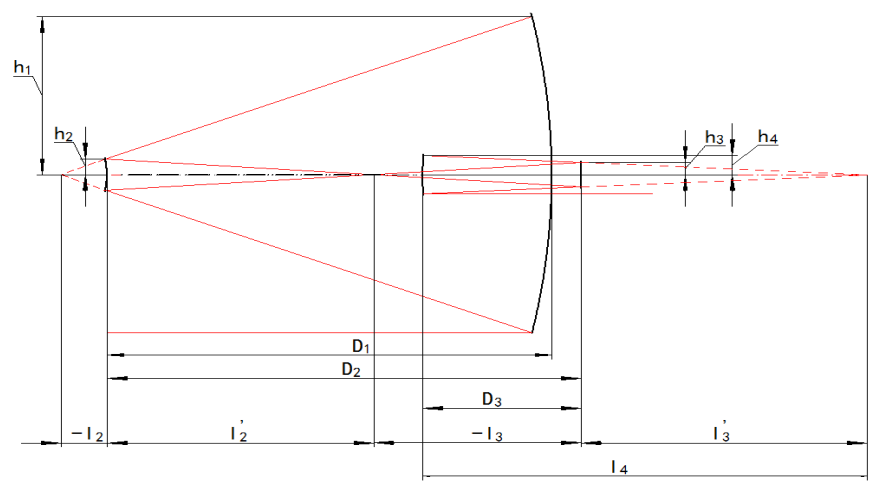

Figure 1. Initial structure of the optical system. 


$$
\beta_{2}=l_{3}^{\prime} / l_{3}=u_{3} / u_{3}^{\prime}
$$

For reflective systems $n_{1}=n_{2}^{\prime}=n_{3}=n_{4}^{\prime}=1, \quad n_{1}^{\prime}=n_{2}=n_{3}^{\prime}=n_{4}=-1$. For afocal systems, the object and the image are located at infinity, then $l_{1}=\infty, l_{4}^{\prime}=$ $\infty, u_{1}=0, u_{4}^{\prime}=0$. According to the requirements, the system compression ratio $\tau=\alpha_{1} \alpha_{2} \alpha_{3}$, the field of view $\omega=200 \mu \mathrm{rad}, h_{1}=100 \mathrm{~mm}, J=-h_{1} \omega$. The aperture stop is located before the primary mirror, then $x_{1}=0, y_{1}=0$. Considering the manufacture and test of $\mathrm{M} 1$, the main mirror diameter is $200 \mathrm{~mm}$, and the off-axis quantity is more than $100 \mathrm{~mm}$ to remove the central obscuration, then the radius of curvature of $R_{1}$ is set to be $1000 \mathrm{~mm}$ to render a $F$ number close to 1.2. Combined with the Gaussian formula, it can be derived as follows:

$$
\begin{gathered}
u_{1}^{\prime}=\frac{2 h_{1}}{R_{1}}, u_{2}=u_{1}^{\prime}, u_{2}^{\prime}=\frac{2 h_{1}}{R_{1} \beta_{1}}, u_{3}=u_{2}^{\prime}, u_{3}^{\prime}=\frac{2 h_{1}}{R_{1} \beta_{1} \beta_{2}}, u_{4}=u_{3}^{\prime} \\
y_{2}=\left(\frac{R_{1}}{2}-\frac{R_{1} \tau}{2 \alpha_{2} \alpha_{3}}\right) \omega, y_{3}=\frac{R_{1}\left[\alpha_{3}\left(\alpha_{2}-\beta_{1}+\alpha_{2} \beta_{1}\right)-\tau\right] \omega}{2 \alpha_{3}}, \\
y_{4}=-\frac{R_{1}\left[\tau-\alpha_{2} \alpha_{3}\left(1+\beta_{1}\right)+\beta_{1}\left(\alpha_{3}-\beta_{2}+\alpha_{3} \beta_{2}\right)\right] \omega}{2} \\
R_{2}=\frac{R_{1} \alpha_{1} \beta_{1}}{1+\beta_{1}}, R_{3}=\frac{R_{1} \alpha_{1} \alpha_{2} \beta_{1} \beta_{2}}{1+\beta_{2}}, R_{4}=R_{1} \alpha_{1} \alpha_{2} \alpha_{3} \beta_{1} \beta_{2} \\
D_{1}=\frac{R_{1}\left(1-\alpha_{1}\right)}{2}, D_{2}=\frac{R_{1} \alpha_{1} \beta_{1}\left(1-\alpha_{2}\right)}{2}, D_{3}=\frac{R_{1} \alpha_{1} \alpha_{2} \beta_{1} \beta_{2}\left(1-\alpha_{3}\right)}{2}
\end{gathered}
$$

It can be seen from Equations (8) and (9) that the structural form of the off-axis four-reflex optical system is completely determined by $\alpha_{1}, \alpha_{2}, \alpha_{3}, \beta_{1}, \beta_{2}$. And the system structure is characterized byan intermediate image plane between $\mathrm{M}_{3}$ and $\mathrm{M}_{4}$. The range of the structural parameters is shown in Table 2. It should be noted that in order to improve the manufacturability of the telescope, the size of the reflective element should be controlled, and during the optimization process, the majority of the power is settled by primary mirror and secondary mirror, and the absolute value of the $\beta_{2}$ is close to 1 .

Substituting (6) (7) into the Seidel aberration coefficient expression (10), and finally the Seidel aberration can be solved (11):

$$
\begin{aligned}
& W_{i}=\frac{\Delta u_{i}}{\Delta\left(1 / n_{i}\right)} \Delta \frac{u_{i}}{n_{i}}, P_{i}=W_{i} \frac{\Delta u_{i}}{\Delta\left(1 / n_{i}\right)}, \Pi_{i}=\frac{\Delta\left(n_{i} u_{i}\right)}{n_{i} n_{i}^{\prime}}, \Phi_{i}=\frac{1}{h_{i}} \Delta \frac{u_{i}}{n_{i}}, K_{i}=\frac{k_{i}}{R_{i}^{3}} \Delta n_{i}(10) \\
& S_{I}=\sum_{i=1}^{4} h_{i} P_{i}+\sum_{i=1}^{4} h_{i}^{4} K_{i} \\
& S_{I I}=\sum_{i=1}^{4} y_{i} P_{i}+J \sum_{i=1}^{4} W_{i}+\sum_{i=1}^{4} h_{i}^{3} y_{i} K_{i} \\
& \left\{S_{I I I}=\sum_{i=1}^{4} \frac{y_{i}^{2}}{h_{i}} P_{i}-2 J \sum_{i=1}^{4} \frac{y_{i}}{h_{i}} W_{i}+J^{2} \sum_{i=1}^{4} \Phi_{i}+\sum_{i=1}^{4} h_{i}^{2} y_{i}^{2} K_{i}\right. \\
& S_{I V}=\sum_{i=1}^{4} \frac{\Pi_{i}}{h_{i}} \\
& S_{V}=\sum_{i=1}^{4} \frac{y_{i}^{3}}{h_{i}^{3}} P_{i}-3 J \sum_{i=1}^{4} \frac{y_{i}^{2}}{h_{i}^{2}} W_{i}+J^{2} \sum_{i=1}^{4} \frac{y_{i}}{h_{i}}\left(3 \Phi_{i}+\frac{\Pi_{i}}{h_{i}}\right)-J^{3} \sum_{i=1}^{4} \frac{1}{h_{i}^{2}} \Delta\left(\frac{1}{n_{i}^{2}}\right)+\sum_{i=1}^{4} h_{i} y_{i}^{3} K_{i}
\end{aligned}
$$


Table 2. Range of structural parameters.

\begin{tabular}{cc}
\hline Structural parameters & Range \\
\hline$\alpha_{1}$ & $(0,1)$ \\
$\alpha_{2}$ & $(-\infty, 0)$ \\
$\alpha_{3}$ & $(0, \infty)$ \\
$\beta_{1}$ & $(-\infty, 0)$ \\
$\beta_{2}$ & $(-\infty, \infty)$ \\
\hline
\end{tabular}

where $S_{I}, S_{I I}, S_{\text {III }}, S_{\text {IV }}$ and $S_{V}$ represent spherical aberration, coma, astigmatism, field curvature and distortion respectively.

The design variables include $\alpha_{1}, \alpha_{2}, \alpha_{3}, \beta_{1}, \beta_{2}$, and 4 conic coefficients which is $k_{1}, k_{2}, k_{3}, k_{4}$. For four-mirror afocal optical system, the requirement for field curvature can be relaxed due to the small FOV. Because the diameter of the exit pupil is only $5 \mathrm{~mm}$, the size of tertiary mirror and quaternary mirror are relatively small accordingly. To simplify the system complexity, the tertiary mirror and quaternary mirror are spherical, ie $k_{3}=0, k_{4}=0$, then the number of design variable reduces seven. In order to eliminate system spherical aberration, coma, astigmatism, curvature of field and distortion, there are still redundant degrees of freedom to control the feature of optical structure, such as the size of the secondary mirror, the position of the exit pupil, the mirror intervals and the working distance. The initial structural design results are shown in Figure 2.

\section{Optimization of the Optical System}

Based on the initial structure, an offset is introduced to the apertures to eliminate the center obscuration, and the tertiary mirror and quaternary mirror are tilted to ensure that the system has available exit pupil. In the optimization, the wave aberration is optimized for the scientific field of view, and the MTF is optimized for the field of regard to ensure good image quality after being connected with imaging system of the subsequent design. During the optimization, the structural layout is adjusted reasonably, the entrance pupil is adjusted to the front of the telescope, and the interval between primary and secondary and the size of secondary mirror are controlled. At the same time, the conic coefficients of the secondary mirror are constrained to suppress the asphericity of the secondary mirror. In addition, the high stray light specification leads to an extremely high requirement on the surface roughness of the optical elements. The position and size of the mechanical structure such as baffles, field stop and aperture stop should be considered to reduce the stray light generated by the non-imaging beam. The optical system structure after optimization is shown in Figure 3, and the parameters are shown in Table 3.

\section{Image Quality Analysis}

In the scientific field of view, take $(0,0),(5.6 \mu \mathrm{rad}, 0),(8 \mu \mathrm{rad}, 0),(0,5.6 \mu \mathrm{rad})$, 


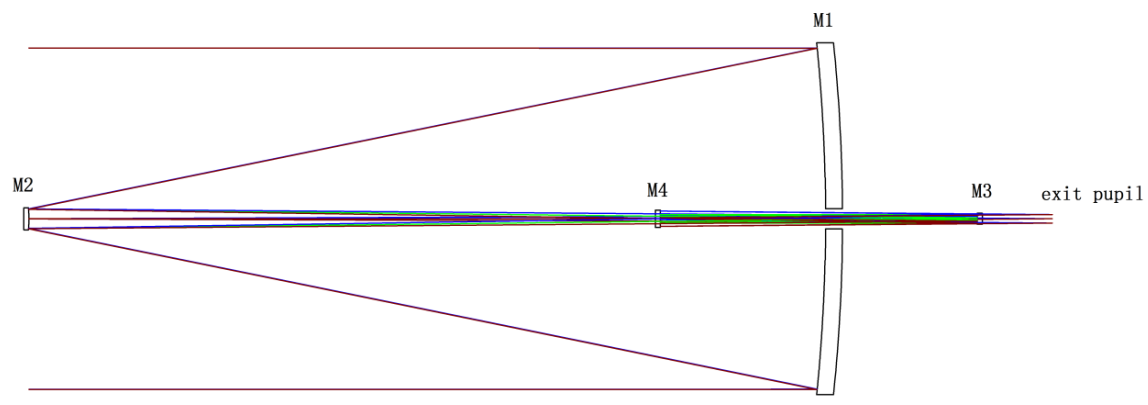

Figure 2. Initial structural design results.

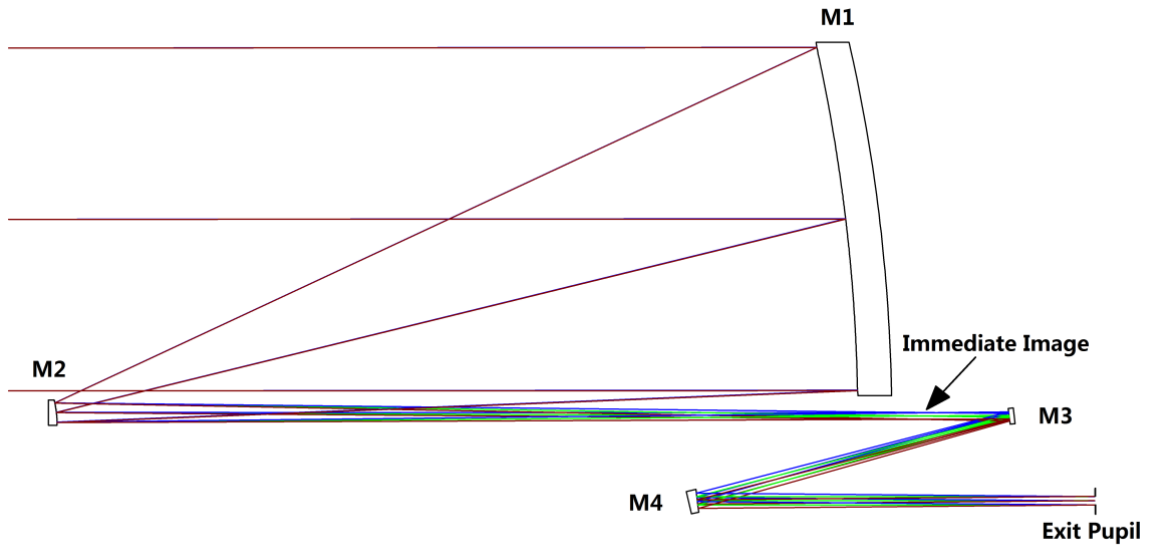

Figure 3. Optical system structure.

Table 3. Structural parameters after optimization.

\begin{tabular}{cccc}
\hline Mirrors & Radius $/ \mathrm{mm}$ & Interval $/ \mathrm{mm}$ & Conic Type \\
\hline Primary & -1000 & -470.5 & Ellipsoid \\
Secondary & -60.53 & 535.58 & Ellipsoid \\
Tertiary & -988.88 & -205.02 & \\
Quaternary & -460.88 & -216.91 & \\
\hline
\end{tabular}

$(0,8 \mu \mathrm{rad}),(0,-5.6 \mu \mathrm{rad}),(-8 \mu \mathrm{rad}, 0)$ as typical fields for image quality analysis. The wavefront errors over the fields are shown in Figure 4. The wavefront consistency of each field is excellent. The maximum root mean square (RMS) wavefront error is less than $0.007 \lambda$, and peak-to-vally (PV) is less than $0.03 \lambda(\lambda=1064$ $\mathrm{nm})$. The MTF curves are shown in Figure 5, and the optical path difference (OPD) of is shown in Figure 6. The image quality is close to the diffraction limit.

\section{TTL Coupled Noise Caused by Wavefront Error}

After propagation of millions of kilometers the beam received by the telescope is a plane wave with uniform intensity and phase. After beam shrinking, the wavefront error of the telescope is coupled into the optical bench to interfere with the local reference laser beam which is a fundamental mode Gaussian beam. Set the beam waist of Gaussian beam at the exit of the telescope, as shown in Figure 7. 


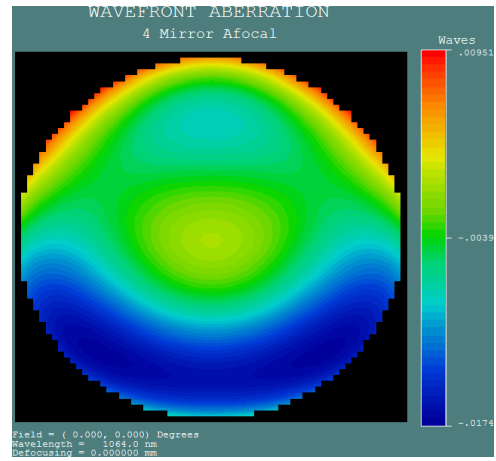

(a)

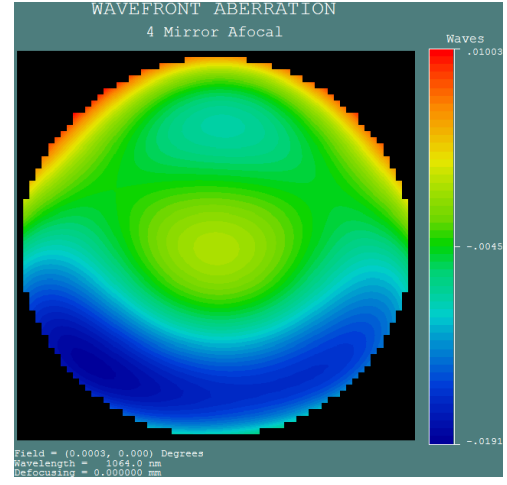

(b)

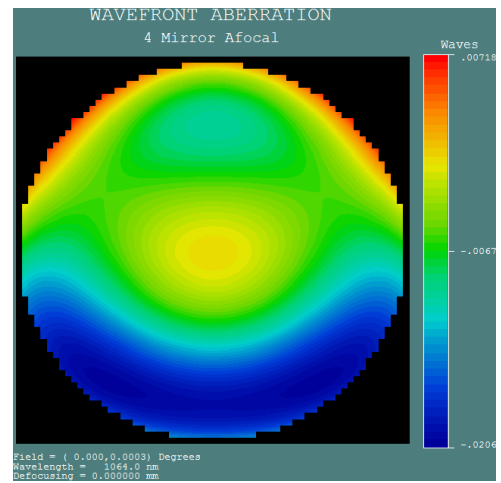

(d)

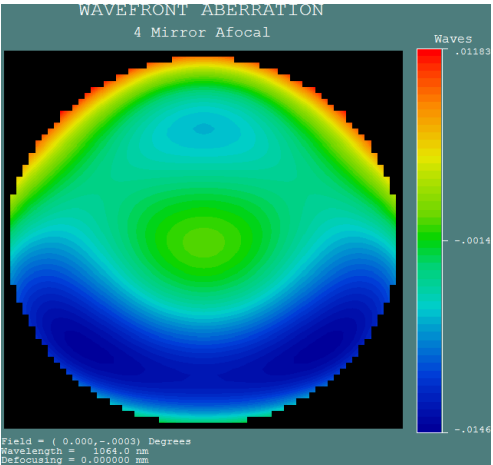

(f)

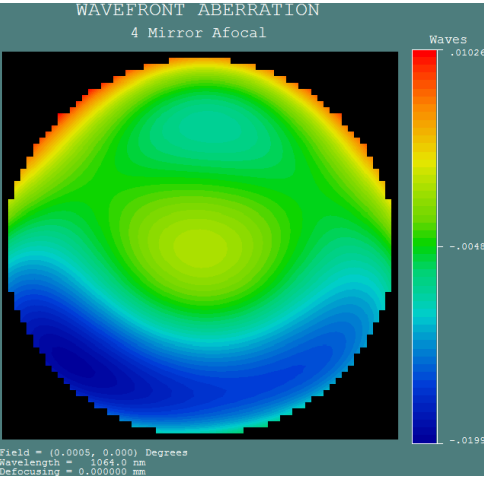

(c)

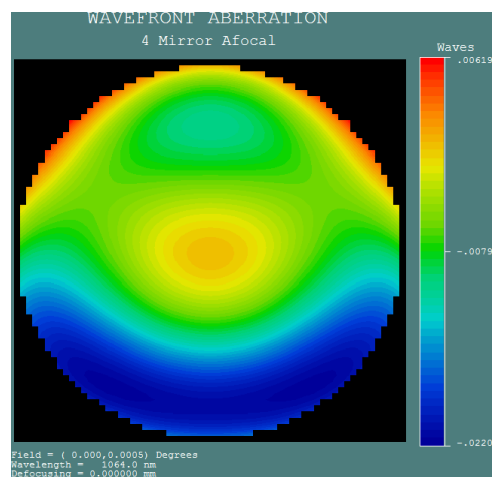

(e)

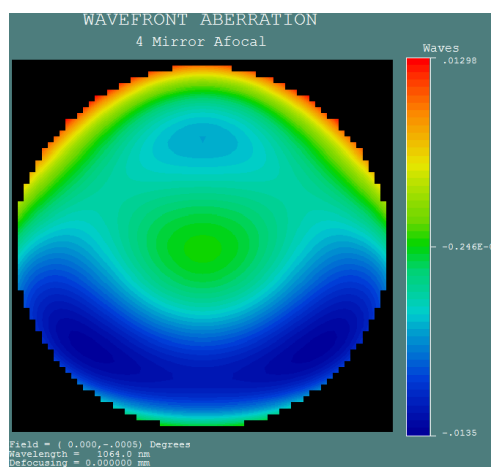

(g)

Figure 4. Wavefronterror over the scientific field of view. (a) Wavefront error of $(0,0)$; (b) Wavefront error of $(5.6 \mu \mathrm{rad}, 0)$; (c) Wavefront error of $(8 \mu \mathrm{rad}$, $0)$; (d) Wavefront error of (0, 5.6 $\mu \mathrm{rad})$; (e) Wavefront error of $(0,8 \mu \mathrm{rad})$. 


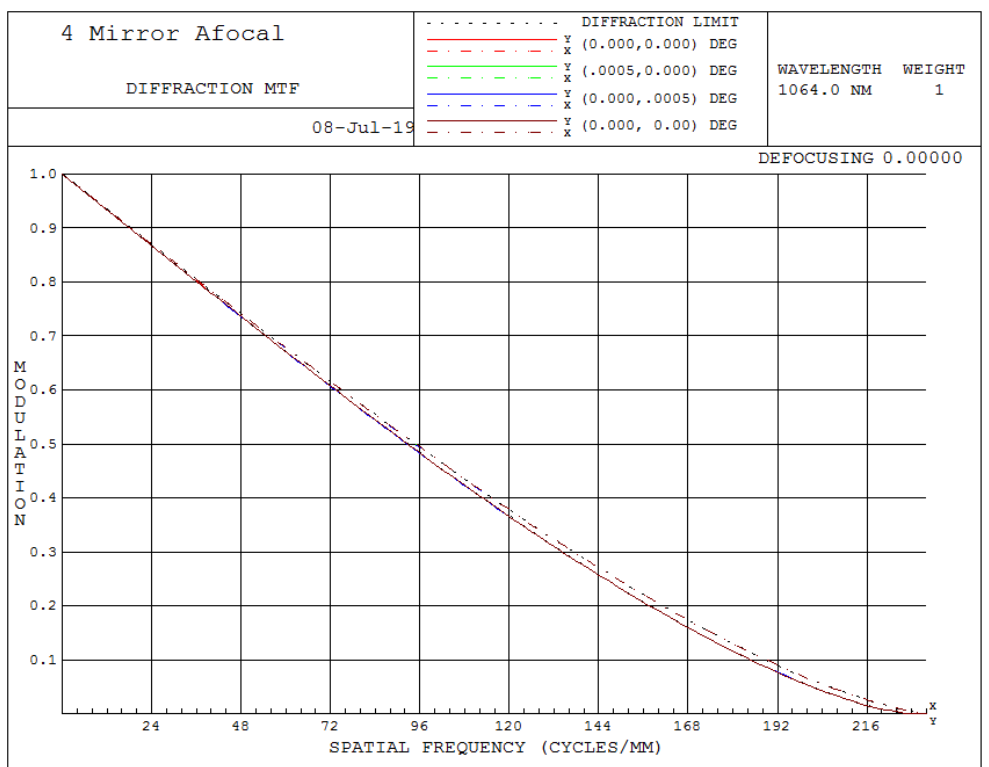

Figure 5. MTF curves.

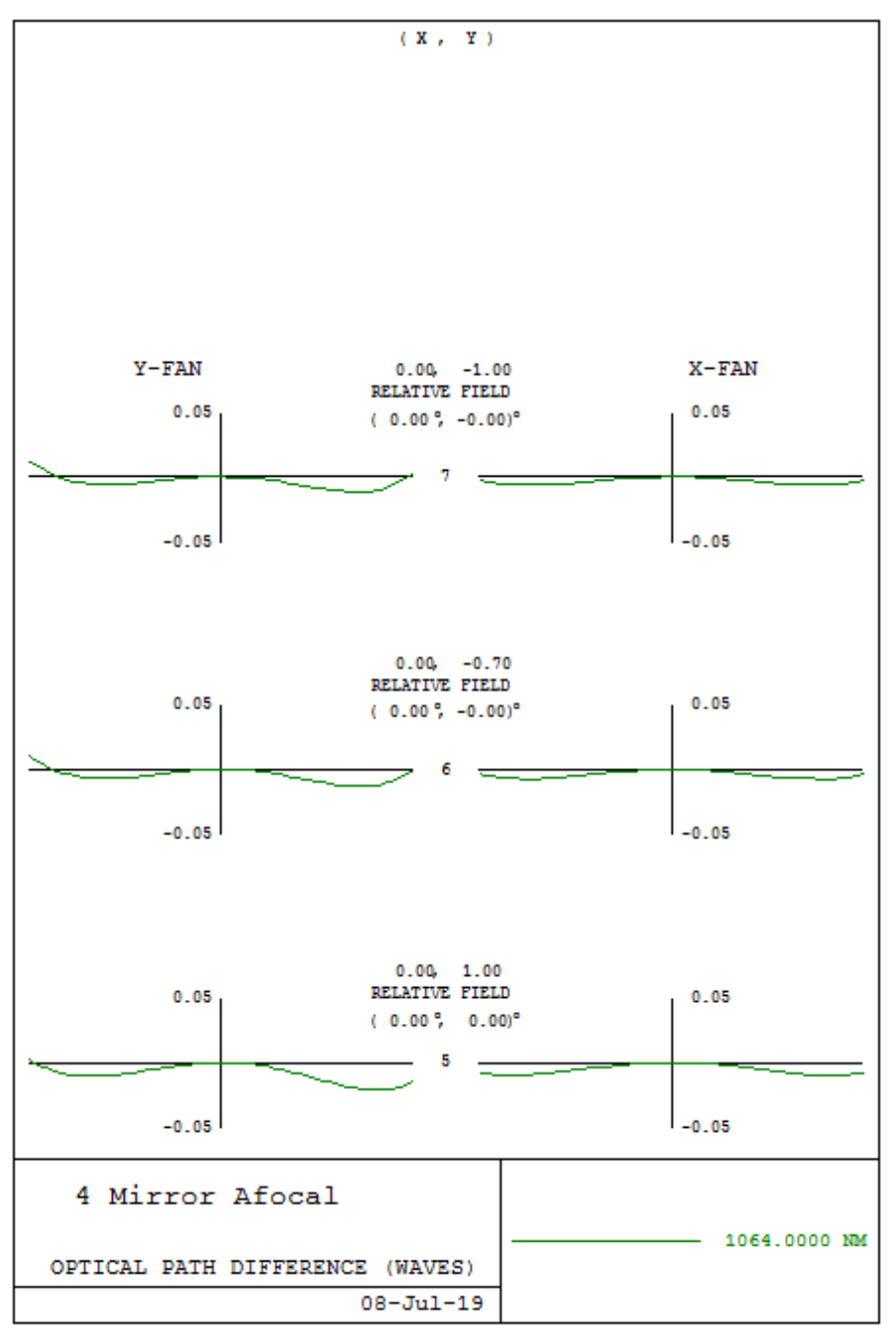

Figure 6. OPD curves. 


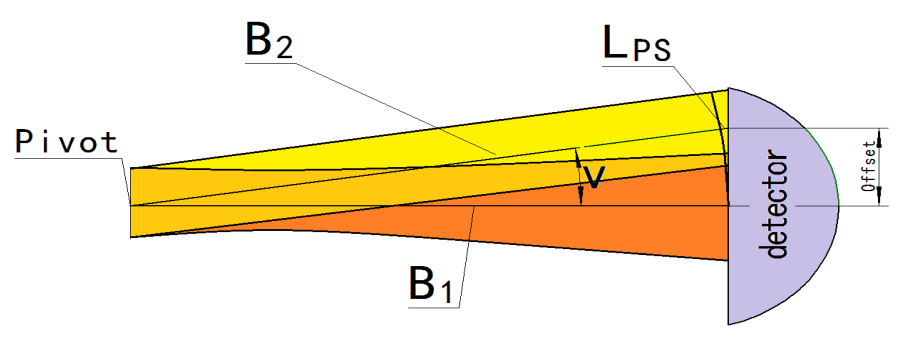

Figure 7. Geometric Schematic of TTL coupling.

During the gravitational wave measurement, the presence of the orbital respiration angle causes the position and pointing of the transmitting end telescope to jitter continuously within the scientific field of view of the receiving end telescope. The beam angle in image space of the receiving end telescope will be within the continuous range of $\pm 8 \mu \mathrm{rad} \times 40= \pm 320 \mu \mathrm{rad}$. Namely, the angle $\mathrm{v}$ in Figure 7 varies within $320 \mu \mathrm{rad}$, which in turn introduces TTL noise. According to LISA's noise budget, in the range of $(-300 \mu \mathrm{rad},+300 \mu \mathrm{rad})$ the absolute value of TTL coupling noise slope should be limited under $25 \mu \mathrm{m} / \mathrm{rad}$.

The phase signal can be solved according to Equation (12), where the complex amplitude of the reference beam is $b_{1}$ and the measuring beam is $b_{2}$. The optical path signal $L_{\mathrm{PS}}$ can be solved by Equation (13).

$$
\begin{gathered}
\left\{\begin{array}{l}
a=\int_{s} d^{2} r B_{1} B_{2}^{*} \\
\Delta \varphi=\arg (a)
\end{array}\right. \\
L_{p s}=\frac{\Delta \varphi}{k}
\end{gathered}
$$

After tilted by angle $v$, the measuring beam containing a wavefront residual $\Delta W$ can be expressed as:

$$
B_{2}=A_{2} \exp \left(-i k(x \sin (v)+z \cos (v))-\omega_{f} t\right) \exp (2 \pi i \Delta W)
$$

The average wavefront residuals of the typical fields as shown in Figure 4 is taken into Equations (12)-(14), and the TTL noise due to the wavefront error of the telescope is obtained through theoretical analysis, as shown in Figure 8(a). Correspondingly, The TTL noise slope is shown in Figure 8(b). Obviously, the absolute value of TTL noise slope within the range of $(-300 \mu \mathrm{rad},+300 \mu \mathrm{rad})$ is less than $0.6 \mu \mathrm{m} / \mathrm{rad}$.

\section{Conclusion}

The inter-satellite interferometry telescope plays an important role of the signal transmission and reception in long arm high precision laser distance measurement. Based on the primary aberration theory, this paper describes the method design of the off-axis four-mirror afocal optical system, for the application of gravitational wave detection. Then the optical system is optimized, with an entrance pupil diameter of $200 \mathrm{~mm}$, a compression ratio of 40 , a scientific field of view of $\pm 8 \mu \mathrm{rad}$, a intermediate image plane and an available exit pupil. Over the 


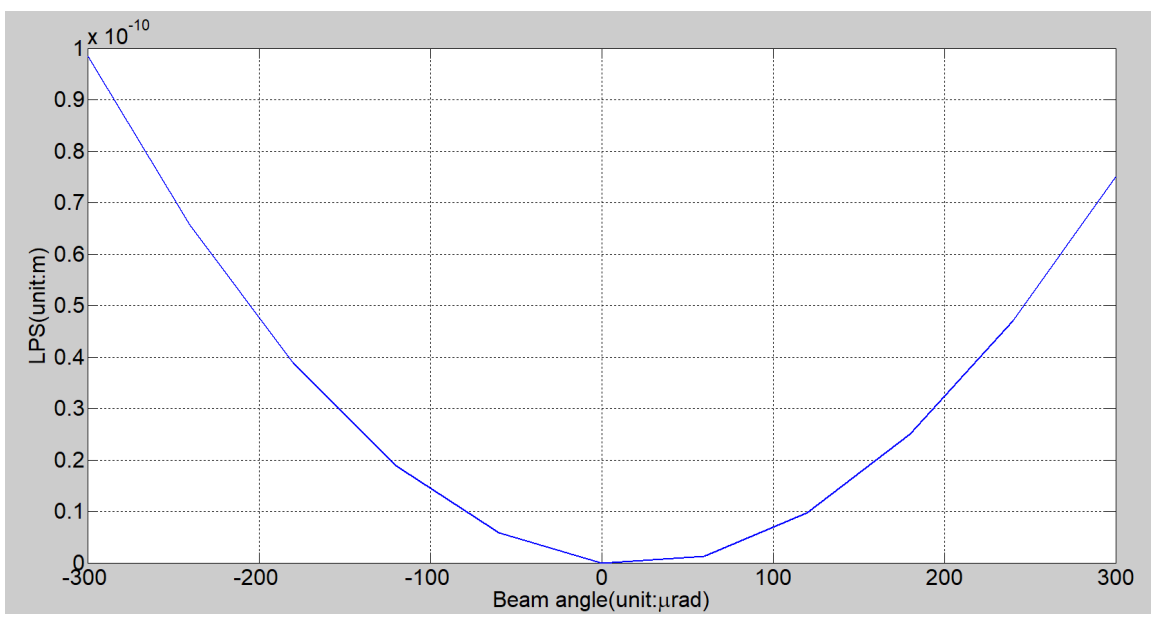

(a)

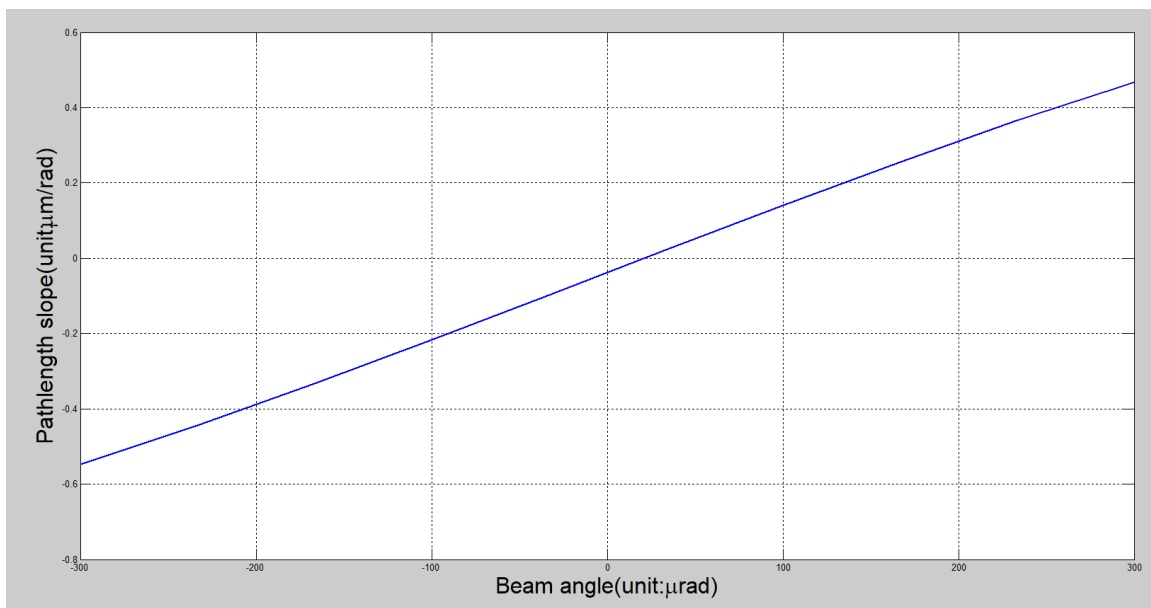

(b)

Figure 8. Analysis of TTL coupling noise. (a) TTL noise caused by wavefronterror; (b) TTL noise change rate caused by wavefront residual.

scientific field of view, the wavefront error RMS value is less than $0.007 \lambda$, and the PV value less than $0.03 \lambda(\lambda=1064 \mathrm{~nm})$. The imaging quality is close to the diffraction limit, and the TTL noise caused by the telescope wavefront residual within the range of $(-300 \mu \mathrm{rad},+300 \mu \mathrm{rad})$ is less than $1 \times 10^{-10} \mathrm{~m}$, whose absolute slope is under $0.6 \mu \mathrm{m} / \mathrm{rad}$, satisfying LISA's requirement of less than 25 $\mu \mathrm{m} / \mathrm{rad}$. The designed optical system meets the special requirements of the gravitational wave detection mission which is different from the traditional imaging system.

\section{Conflicts of Interest}

The authors declare no conflicts of interest regarding the publication of this paper.

\section{References}

[1] Jennrich, O. (2009) LISA Technology and Instrumentation. Classical and Quantum 
Gravity, 26, Article ID: 153001.

[2] Wang, Z., Sha, W., Chen, Z., Kang, Y.-S., Luo, Z.-R., Li, M. and Lin, Y.-P. (2018) Preliminary Design and Analysis of Telescope for Space Gravitational Wave Detection. Chinese Optics, 11, 131-151.

[3] Livas, J.C., Arsenovic, P., Crow, J.A., Hill, P.C., Howard, J.M., Seals, L.T., and Shiri, S. 2013. Telescopes for Space-Based Gravitational Wave Missions. Optical Engineering, 52, Article ID: 091811. https://doi.org/10.1117/1.oe.52.9.091811

[4] Schuster, S., Wanner, G., Tröbs, M. and Heinzel, G. (2015) Vanishing Tilt-to-Length Coupling for a Singular Case in Two-Beam Laser Interferometers with Gaussian Beams. Applied Optics, 54, 1010-1014. https://doi.org/10.1364/ao.54.001010

[5] Schuster, S. (2017) Tilt-to-Length Coupling and Diffraction Aspects in Satellite Interferometry. Doctoral Dissertation, Gottfried Wilhelm Leibniz Universität Hannover.

[6] Wanner, G. (2010) Complex Optical Systems in Space: Numerical Modelling of the Heterodyne Interferometery of LISA Pathfinder and LISA. Doctoral Dissertation, Laser Interferometry \& Gravitational Wave Astronomy, AEI-Hannover, MPI for Gravitational Physics, Max Planck Society.

[7] Wilson, R.N. (2013) Reflecting Telescope Optics II: Manufacture, Testing, Alignment, Modern Techniques. Springer Science \& Business Media.

[8] Xuezhuan, D., Xin, W., Weihua, L. and Yinnian, L. (2008) Design of Four-Mirror Reflective Anastigmat Optic System. Infrared and Laser Engineering, 37, 319-321. 